PoS $\quad \begin{aligned} & \text { PROCEEDINGS } \\ & \text { OF SCIENCE }\end{aligned}$

\title{
Scattering from finite size methods in lattice QCD
}

\section{DESY 09-171 \\ SFB/CPP-09-95 \\ MS-TP-09-21}

\author{
Xu Feng ${ }^{* a, b}$, Karl Jansen ${ }^{a}$ and Dru B. Renner ${ }^{a}$ \\ ${ }^{a}$ NIC, DESY, Platanenallee 6, D-15738 Zeuthen, Germany \\ ${ }^{b}$ Universität Münster, Institut für Theoretische Physik, Wilhelm-Klemm-Strasse 9, D-48149 \\ Münster, Germany \\ E-mail: xu.feng@desy.de
}

Using two flavors of maximally twisted mass fermions, we calculate the S-wave pion-pion scattering length in the isospin $I=2$ channel and the $\mathrm{P}$-wave pion-pion scattering phase in the isospin $I=1$ channel. In the former channel, the lattice calculations are performed at pion masses ranging from $270 \mathrm{MeV}$ to $485 \mathrm{MeV}$. We use chiral perturbation theory at next-to-leading order to extrapolate our results. At the physical pion mass, we find $m_{\pi} a_{\pi \pi}^{I=2}=-0.04385$ (28)(38) for the scattering length. In the latter channel, the calculation is currently performed at a single pion mass of $391 \mathrm{MeV}$. Making use of finite size methods, we evaluate the scattering phase in both the center of mass frame and the moving frame. The effective range formula is employed to fit our results, from which the rho resonance mass and decay width are evaluated.

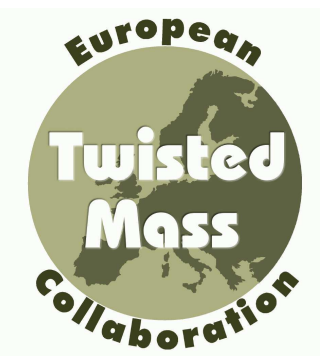

The XXVII International Symposium on Lattice Field Theory - LAT2009

July 26-31 2009

Peking University, Beijing, China

\footnotetext{
*Speaker.
} 


\section{Introduction}

Experimentally, hadron-hadron scattering is an important method to study the strong interactions. Among the various scattering possibilities, pion-pion scattering is the simplest and best understood one due to the fact that the underlying chiral symmetry strongly determines the low energy behavior of the pion-pion scattering amplitude. Despite its simplicity, pion-pion scattering offers us a lot of information on the strong interactions. In the isospin $I=2$ channel, near threshold the S-wave scattering length is used to determine the corresponding low energy constants (LECs) of chiral perturbation theory ( $\chi \mathrm{PT}$ ). In the $I=1$ channel, the prominence of the rho resonance is clearly observed. By measuring the P-wave scattering phase, the parameters for the resonance mass and decay width can be extracted. In the $I=0$ channel, the sigma resonance appears in pion-pion scattering. In contrast to the rho resonance, a precise identification of the sigma resonance remains a great challenge because the large decay width of the sigma causes a strong overlap between it and its background.

Pion-pion scattering is non-perturbative in nature at low energies. Therefore, it should be studied with a non-perturbative method like lattice QCD. In the center-of-mass frame (CMF), a direct lattice QCD determination of the scattering phase is possible by employing Lüscher's finite-size methods $[1,2,3,4,5]$, which establish relations between the discrete energy spectrum in the finite volume and the elastic scattering phase in the infinite volume. In the moving frame (MF), where the total momentum of the pion-pion scattering system is fixed to be a non-zero value, one can evaluate the scattering phase by using the method proposed by Rummukainen and Gottlieb [6], which is an extension of Lüscher's method to MFs. To perform our calculations, we use the $N_{f}=2$ maximally twisted mass fermion ensembles from the European Twisted Mass Collaboration (ETMC). Due to the properties of twisted mass fermions at maximal twist, our calculation is automatically accurate to $O\left(a^{2}\right)$ in the lattice spacing, $a$.

In this paper, we present a calculation of the S-wave pion-pion scattering length in the $I=2$ channel and the $\mathrm{P}$-wave scattering phase in the $I=1$ channel. A calculation of pion-pion scattering in the $I=0$ channel using $2+1$ flavors of domain wall fermions has been reported recently [7]. Although the object of our investigation is simply pion-pion scattering, the approach to study scattering from finite size methods in lattice QCD is universal and can be applied to other meson-meson, meson-baryon and baryon-baryon scattering systems.

\section{2. $I=2$ channel}

In the $I=2$ channel, the lattice calculation is performed in the CMF. As mentioned in the introduction, Lüscher's finite size method relates the energy levels of two pion states in a finite volume to the scattering phase in the infinite volume. For the case of two pions with zero relative three-momentum, this method establishes a relationship between the lowest energy eigenvalue $E_{\pi \pi}^{I=2}$ in a finite box of size $L$ and the corresponding scattering length $a_{\pi \pi}^{I=2}$ [2]:

$$
\delta E_{\pi \pi}^{I=2}=E_{\pi \pi}^{I=2}-2 m_{\pi}=-\frac{4 \pi a_{\pi \pi}^{I=2}}{m_{\pi} L^{3}}\left[1+c_{1} \frac{a_{\pi \pi}^{I=2}}{L}+c_{2}\left(\frac{a_{\pi \pi}^{I=2}}{L}\right)^{2}\right]+O\left(L^{-6}\right),
$$

where $c_{1}=-2.837297$ and $c_{2}=6.375183$ are numerical constants. Thus the above result allows us to convert a lattice determination of the energy shift $\delta E_{\pi \pi}^{I=2}$ into a calculation of $a_{\pi \pi}^{I=2}$. 
To extract the energy shift, $\delta E_{\pi \pi}^{I=2}$, and then the scattering length, $a_{\pi \pi}^{I=2}$, we use the two flavor maximally twisted mass fermion configurations from ETMC $[8,9,10] .{ }^{1}$ The pion masses range from $m_{\pi}=270 \mathrm{MeV}$ to $485 \mathrm{MeV}$. For most of the ensembles, the lattice spacing is $a=0.086 \mathrm{fm}$ and the box size is $L=2.1 \mathrm{fm}$. For the lower pion masses the volume is increased to $L=2.7 \mathrm{fm}$. Additionally, we perform a check for lattice artifacts with a single calculation at a finer lattice spacing of $a=0.067 \mathrm{fm}$. All the results for the scattering length are shown in Fig. 1 .

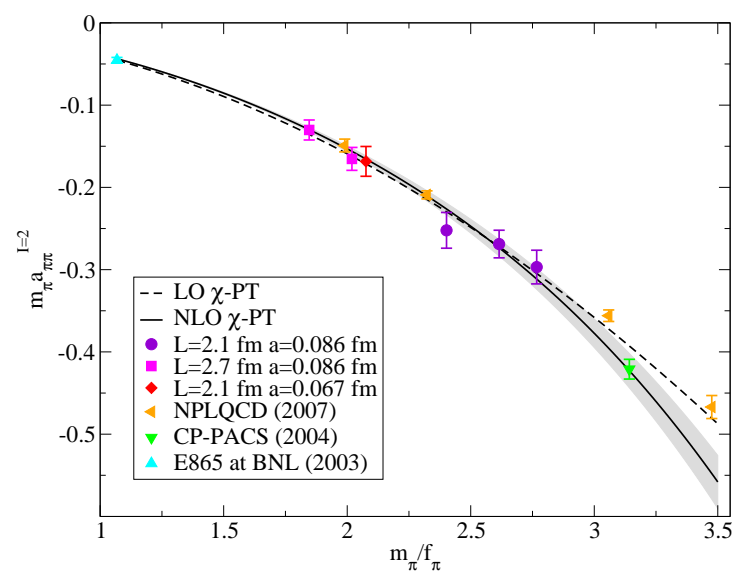

Figure 1: Chiral extrapolation for the $\mathrm{I}=2$ pionpion scattering length. The results in this work are shown together with the lattice calculations of NPLQCD [12, 13] and CP-PACS [14] and the experimental data from E865 at BNL [15].

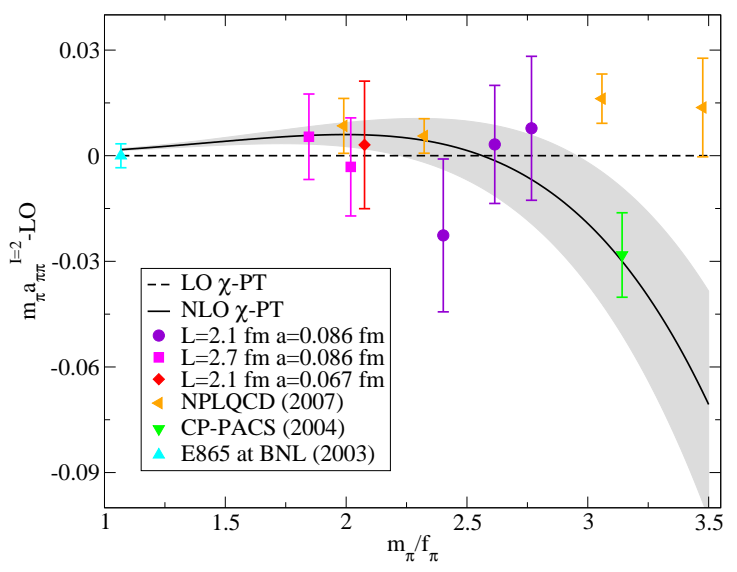

Figure 2: Difference between the lattice calculation of the scattering lengths and the LO $\chi \mathrm{PT}$ prediction. The scattering lengths agree statistically with the LO $\chi \mathrm{PT}$ prediction for pion masses ranging from $m_{\pi}=$ $270 \mathrm{MeV}$ to $485 \mathrm{MeV}$.

The next step is to extrapolate the scattering length to the physical limit. Here, we make use of NLO $\chi$ PT for the pion-pion scattering length, which has recently been studied in the twisted mass case [16]. The $\chi \mathrm{PT}$ fit curves are shown in Fig. 1. In the same figure, we also provide a comparison to the lattice results of NPLQCD $[12,13]$ and CP-PACS [14] and the experimental data from E865 at BNL [15]. We find general agreement between our calculation and the results of NPLQCD at similar pion masses. Additionally, we find agreement with the experimental determination of $m_{\pi} a_{\pi \pi}^{I=2}$. To highlight the impact of the NLO terms in the $\chi \mathrm{PT}$ description of the pion mass dependence of $m_{\pi} a_{\pi \pi}^{I=2}$, we show the difference between the lattice calculations of the scattering length and the LO $\chi$ PT prediction in Fig. 2. We find that the scattering lengths statistically agree with the LO $\chi$ PT result for all lattice calculations with $m_{\pi}<500 \mathrm{MeV}$. Accordingly, the NLO $\chi$ PT functional form provides a reasonable description of the lattice results in the same region of $m_{\pi}$. At the physical pion mass, we obtain the final results

$$
m_{\pi} a_{\pi \pi}^{I=2}=-0.04385(28)(38) \quad \text { and } \quad l_{\pi \pi}^{I=2}\left(\mu=f_{\pi, \mathrm{phy}}\right)=4.65(.85)(1.07)
$$

where the first error is statistical and the second is our estimate of several systematic effects. For more details, we refer the reader to our recent paper [17].

\footnotetext{
${ }^{1}$ The role of the neutral pion in our setup is discussed in some detail in Ref. [11]
} 


\section{3. $I=1$ channel}

In the $I=1$ channel, the rho meson decays into two pions in the P-wave. As the case of Swave, one can make use of finite size methods to calculate the P-wave scattering phase. However, the extraction of the rho resonance from the scattering phase is non-trivial for several reasons. First, only when the pion masses are light enough to satisfy the requirement of $m_{\pi}<m_{\rho} / 2$, is it possible for the rho to decay into two pions. Second, the standard form of Lüscher's method is derived to address the elastic scattering process, so the interesting energy spectrum should be smaller than $4 m_{\pi}$ to avoid the inelastic scattering. Third, because of the finite volume, the energy spectrum of pion-pion scattering states is discrete, which translates into scattering phases at discrete energies. Therefore, an analytic expression of the scattering phase is required to describe its dependence on the energy spectrum. Usually, one employs the effective range formula to meet this demand:

$$
\tan \delta_{1}(k)=\frac{g_{\rho \pi \pi}^{2}}{6 \pi} \frac{k^{3}}{E_{C M}\left(M_{R}^{2}-E_{C M}^{2}\right)}, \quad k=\sqrt{E_{C M}^{2} / 4-m_{\pi}^{2}},
$$

where $\delta_{1}(k)$ is a P-wave scattering phase in the $I=1$ channel and $E_{C M}$ is the center-of-mass energy. In the MF, $E_{C M}$ is simply given by $E_{C M}^{2}=E^{2}-\vec{P}^{2}$, where $E$ is the discrete energy eigenvalue and $\vec{P}$ is the total momentum of the MF. ${ }^{2}$ Thus, in the effective range formula, only two parameters are undetermined, $M_{R}$ and $g_{\rho \pi \pi}$, where $M_{R}$ denotes the resonance mass and $g_{\rho \pi \pi}$ is the effective $\rho \rightarrow \pi \pi$ coupling constant, which largely determines the size of resonance decay width:

$$
\Gamma_{R}=\frac{g_{\rho \pi \pi}^{2}}{6 \pi} \frac{k^{3}}{M_{R}^{2}}, \quad k=\sqrt{M_{R}^{2} / 4-m_{\pi}^{2}} .
$$

By fitting the discrete scattering phases to the effective range formula, one can evaluate the parameters $M_{R}$ and $g_{\rho \pi \pi}$ and then determine $\Gamma_{R}$. Conversely, by using the latest PDG [18] values of $m_{\pi}=139.5702(4) \mathrm{MeV}, M_{\rho}=775.49(34) \mathrm{MeV}$ and $\Gamma_{\rho}=149.1(8) \mathrm{MeV}$, one can also evaluate $g_{\rho \pi \pi}=5.98(2)$ at the physical pion mass.

With the effort required to simulate with light up and down quark masses, the condition of $m_{\pi}<m_{\rho} / 2$ has been satisfied by only a few lattice calculations [19, 20, 21]. So far, all these studies concentrated on one or two scattering phases for each ensemble. Since the effective range formula carries two unknown parameters, more scattering phases are needed for a precise fit. To accomplish this goal, a natural way is to calculate the energy spectrum of the higher excited states. In order to isolate the ground state and the first excited state, we set up a $2 \times 2$ correlation matrix

$$
C_{2 \times 2}(t)=\left(\begin{array}{cc}
\left\langle(\pi \pi)^{\dagger}(t)(\pi \pi)(0)\right\rangle & \left\langle(\pi \pi)^{\dagger}(t) \rho(0)\right\rangle \\
\left\langle\rho^{\dagger}(t)(\pi \pi)(0)\right\rangle & \left\langle\rho^{\dagger}(t) \rho(0)\right\rangle
\end{array}\right),
$$

where the interpolating operator $(\pi \pi)(t)$ has the same quantum numbers, $J^{P C}=1^{--}$, as the interpolating operator $\rho(t)$. After diagonalization of the matrix in 3.1, we obtain the energy eigenvalues of the ground state and the first excited state, $E^{n}(n=1,2)$, and then convert them into the scattering phases. More ambitiously, constructing a $N \times N$ matrix allows us to look at even higher excited states. However, the realistic computation of the scattering phase at higher energy, $E^{n}(n>2)$,

\footnotetext{
${ }^{2}$ To reduce lattice discretization effects, we use the relation $\cosh \left(E_{C M}\right)=\cosh (E)-2 \sin ^{2}(P / 2)$ instead.
} 
remains a challenge due to the poor signal-to-noise ratio and the restriction of $E^{n}<4 m_{\pi}$. Another way to determine the scattering phase at more energies is to perform a lattice calculation in the MF. In our case, we use a MF with a total momentum $\vec{P}=\vec{e}_{3} 2 \pi / L$, which provides us another two scattering phase points. In principle, by performing the lattice calculation in the MF with other total momenta, for example $\vec{P}=\left(\vec{e}_{1}+\vec{e}_{2}\right) 2 \pi / L$, it is possible for us to collect even more points. However, we must be careful in choosing the MF because in some MFs the ground state and the first excited state are nearly degenerate and isolating them becomes very difficult.

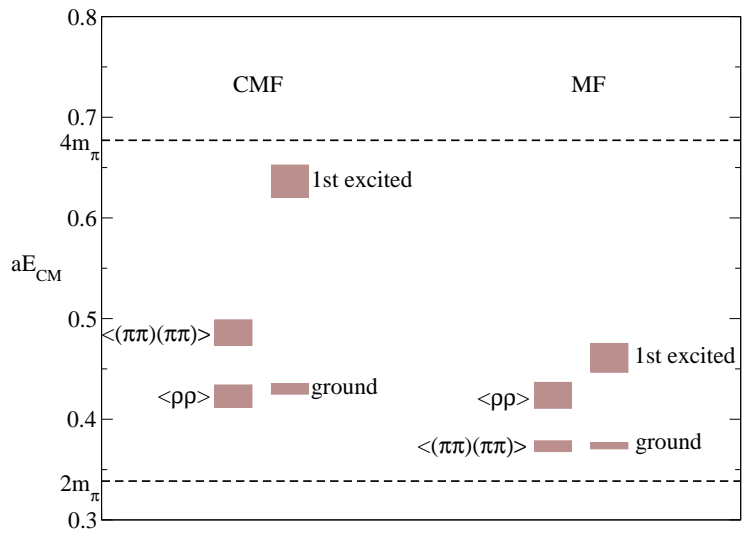

Figure 3: Center-of-mass energies in the CMF and MF. The energies evaluated from the correlation matrix are compared with the ones evaluated from the diagonal matrix elements.

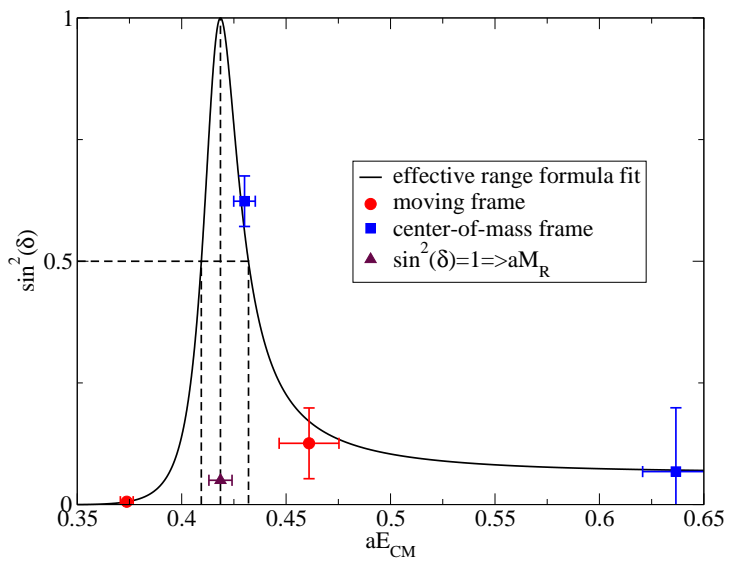

Figure 4: Four scattering phases calculated on the lattice together with the effective range formula fit. At the position where the scattering phase passes $\pi / 2$, the resonance mass $M_{R}$ is determined.

In this work, both the calculations in the CMF and MF are performed by using $N_{f}=2$ dynamical maximally twisted mass fermions. The corresponding lattice parameters are $m_{\pi}=391 \mathrm{MeV}$, $a=0.086 \mathrm{fm}, L=2.1 \mathrm{fm}$ and $m_{\pi} / m_{\rho}=0.4$. By diagonalizing the $2 \times 2$ matrix in 3.1, the $E_{C M}$ of the ground state and the first excited state are evaluated and shown in Fig. 3. In order to investigate the effect of diagonalization, we also perform a study that utilizes only the diagonal matrix elements. We see that in the CMF, there is a strong mixing between the ground state and the first excited state in $\left\langle(\pi \pi)^{\dagger}(t)(\pi \pi)(0)\right\rangle$. While in the MF, a similar situation happens to $\left\langle\rho^{\dagger}(t) \rho(0)\right\rangle$. So no operator safely provides us the ground state energy in both frames. Therefore, introducing the diagonalization method to the calculation of rho decay becomes essential.

As shown in Fig. 3, all the four values of $E_{C M}$ are smaller than $4 m_{\pi}$. Unlike continuum QCD, twisted mass LQCD violates the symmetries of isospin and parity. As a result, it is possible for the rho to decay into three pions, which means that at non-zero lattice spacing the upper bound of the elastic scattering region is lowered to $3 m_{\pi}$. Additionally, the isospin symmetry breaking causes a mixing between the $I=1$ channel and the possible $I=0$ and $I=2$ channels. Thus, a corresponding modification would be required to adapt Lüscher's method to the isospin mixing case. In Ref. [17], a significant effort was made by us to attempt to find these effects in the $I=2$ channel, but no compelling evidence was found. However, the effects of isospin violation in the $\mathrm{I}=1$ channel are expected to be more severe [16]. Just for the purposes of these proceedings, we assume that such effects are small. Therefore, we convert all the four $E_{C M}$ values into the corresponding scattering phases using the normal method. As in Ref. [17], we will eventually examine the effects 


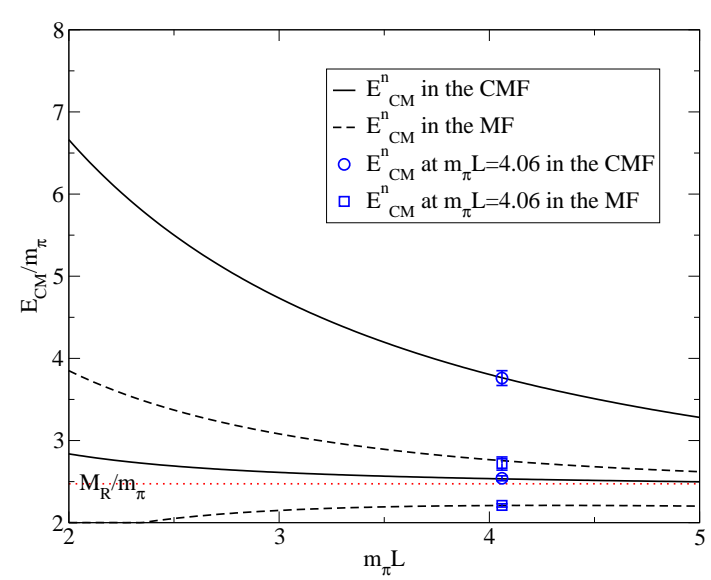

Figure 5: $L$ dependence of $E_{C M}^{n}(\mathrm{n}=1,2)$ at a pion mass of $m_{\pi}=391 \mathrm{MeV}$, using values of $M_{R}$ and $g_{\rho \pi \pi}$ in Eq. 3.2.

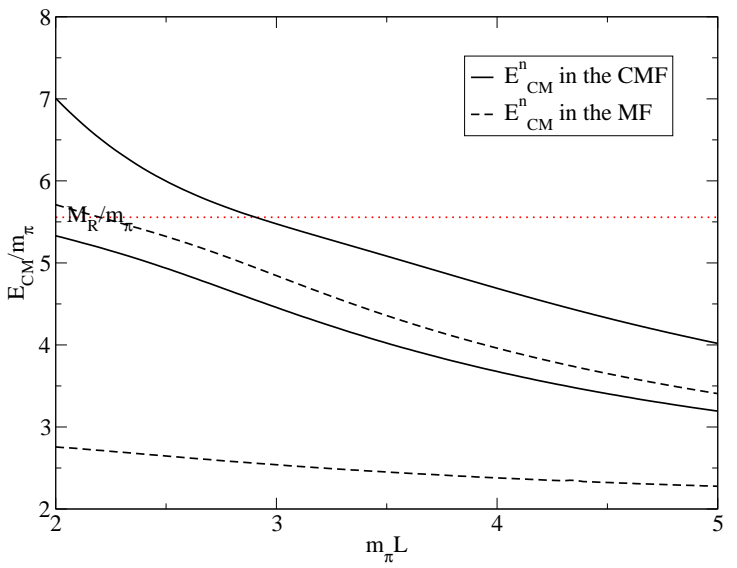

Figure 6: $L$ dependence of $E_{C M}^{n}(\mathrm{n}=1,2)$ in the physical limit, defined by the latest PDG [18] values of $M_{R}$ and $g_{\rho \pi \pi}$.

of parity breaking carefully and complement the current calculation with a calculation at a finer lattice spacing to explicitly check for any strong lattice artifacts for $I=1$.

The results for the scattering phase are shown in Fig. 4 together with the effective range formula fit. At the position where the scattering phase passes $\pi / 2$, the resonance mass $M_{R}$ is determined. Additionally, the values of $g_{\rho \pi \pi}$ and $\Gamma_{R}$ are also evaluated from the fit. Our final results are

$$
a M_{R}=0.4186(56), \quad g_{\rho \pi \pi}=6.16(48) \quad \text { and } \quad a \Gamma_{R}=0.0217(44) .
$$

Here, our result for $g_{\rho \pi \pi}$ at $m_{\pi}=391 \mathrm{MeV}$ agrees statistically with that at the physical pion mass, which hints that the pion mass dependence of $g_{\rho \pi \pi}$ might be weak. However, we can not make any strong statements here since our calculation of rho decay is only performed at one pion mass and our errors are rather large. To determine the chiral limit of $g_{\rho \pi \pi}$ and $M_{R}$, we will perform our calculation at smaller pion masses.

Usually one takes the ground state energy $E_{C M}^{1}$ in the CMF as the rho mass. This is correct in the case $m_{\pi} \geq m_{\rho} / 2$ where the rho is still a stable particle and $E_{C M}^{1}$ has only an exponentially suppressed $L$ dependence. However, when $m_{\pi}$ becomes smaller the rho becomes unstable and $E_{C M}^{1}$ begins to depend more strongly on $L$. As an example, we calculate $E_{C M}(L)$ by combining Lüscher's formula and the effective range formula with the parameters $M_{R}$ and $g_{\rho \pi \pi}$ given in Eq. 3.2. As shown in Fig. 5, the $L$ dependence of the lowest level is visible but still weak at a pion mass of $m_{\pi}=391 \mathrm{MeV}$. Decreasing $m_{\pi}$ further, more phase space becomes available for the rho to decay into two pions. Thus, assuming $g_{\rho \pi \pi}$ is roughly constant, the decay width becomes larger and the lowest level in the CMF begins to look more like a scattering state. Fig. 6 shows $E_{C M}(L)$ in the extreme limit at the physical pion mass. There we see that $E_{C M}^{1}$ drops so rapidly with $L$ that it bears no resemblance to a stable state with mass $M_{R}$.

\section{Conclusions}

We have calculated the $\mathrm{S}$-wave pion-pion scattering length in the $I=2$ channel and the P-wave 
pion-pion scattering phase in the $I=1$ channel using $N_{f}=2$ maximally twisted mass fermions. In the former channel, the pion masses ranged from $270 \mathrm{MeV}$ to $485 \mathrm{MeV}$. Using $\chi \mathrm{PT}$ at NLO, we extrapolated our results for the scattering length to the physical limit, where we found $m_{\pi} a_{\pi \pi}^{I=2}=$ $-0.04385(28)(38)$ and $l_{\pi \pi}^{I=2}\left(\mu=f_{\pi \text {,phy }}\right)=4.65(.85)(1.07)$. In the latter channel, we performed a calculation at $m_{\pi}=391 \mathrm{MeV}, a=0.086 \mathrm{fm}$ and $m_{\pi} / m_{\rho}=0.4$. Making use of finite size methods, we evaluated the scattering phase at four energies, to which we fit the effective range formula and found the results $a M_{R}=0.4186(56), g_{\rho \pi \pi}=6.16(48)$ and $a \Gamma_{R}=0.0217(44)$.

\section{Acknowledgments}

This work was supported by the DFG project Mu 757/13 and the DFG Sonderforschungsbereich / Transregio SFB/TR9-03. We thank G. Herdoiza, A. Shindler, C. Urbach and M. Wagner for valuable suggestions and assistance. X. Feng would like to thank C. Liu, G. Münster, N. Ishizuka and A. Walker-Loud for helpful communications. The computer time for this project was made available by the John von Neumann Institute for Computing on the JUMP and JUGENE systems in Jülich. We also thank the staff of the computer center in Zeuthen for their technical support.

\section{References}

[1] M. Luscher. Commun. Math. Phys., 104:177, 1986.

[2] M. Luscher. Commun. Math. Phys., 105:153-188, 1986.

[3] Martin Luscher and Ulli Wolff. Nucl. Phys., B339:222-252, 1990.

[4] Martin Luscher. Nucl. Phys., B354:531-578, 1991.

[5] Martin Luscher. Nucl. Phys., B364:237-254, 1991.

[6] Steven A. Gottlieb and K. Rummukainen. Nucl. Phys. Proc. Suppl., 47:819-822, 1996.

[7] Qi Liu. arXiv:0910.2658, 2009.

[8] Ph. Boucaud et al. Phys. Lett., B650:304-311, 2007.

[9] Petros Dimopoulos et al. arXiv:0810.2873, 2008.

[10] Carsten Urbach. PoS, LAT2007:022, 2007.

[11] P. Dimopoulos, R. Frezzotti, C. Michael, G. C. Rossi, and C. Urbach. arXiv:0908.0451, 2009.

[12] S. R. Beane, P. F. Bedaque, K. Orginos, and M. J. Savage. Phys. Rev., D73:054503, 2006.

[13] Silas R. Beane et al. Phys. Rev., D77:014505, 2008.

[14] T. Yamazaki et al. Phys. Rev., D70:074513, 2004.

[15] S. Pislak et al. Phys. Rev., D67:072004, 2003.

[16] Michael I. Buchoff, Jiunn-Wei Chen, and Andre Walker-Loud. Phys. Rev., 79:074503, 2009.

[17] Xu Feng, Karl Jansen, and Dru B. Renner. arXiv:0909.3255, 2009.

[18] C. Amsler et al. Phys. Lett., B667:1, 2008.

[19] S. Aoki et al. Phys. Rev., D76:094506, 2007.

[20] M. Gockeler et al. arXiv:0810.5337, 2008.

[21] K. Jansen, C. McNeile, C. Michael, and C. Urbach. arXiv:0906.4720, 2009. 